\title{
Revascularização do miocárdio com emprego da artéria radial: estudo clínico e angiográfico seqüencial
}

\author{
Francisco Diniz Affonso da COSTA*, Robinson POFFO*, Maria Adriana Costa LIMA*, Djalma Luiz \\ FARACO*, Fábio S. SALLUM ${ }^{\star}$, Elson Cox Cruz OLIVEIRA*, Iseu Affonso da COSTA*
}

COSTA, F. D. A.; POFFO, R.; LIMA, M. A. C.; FARACO, D. L.; SALLUM, F. S.; OLIVEIRA, E. C. C.; COSTA, I. A. - Revascularização do miocárdio com emprego da artéria radial: estudo clínico e angiográfico seqũencial. Rev. Bras. Cir. Cardiovasc., 10 (4): 165-174, 1995.

RESUMO: De 8/4/94 a 28/2/95, 32 pacientes foram submetidos a revascularização do miocárdio (RM) com a utilização da artéria radial (RA) como parte do procedimento. Esse número representou $17,3 \%$ da experiência global com RM neste periodo. Vinte e três pacientes eram masculinos e a média de idades foi de 56,8 anos ( 41 a 74 anos). A RA foi utilizada para revascularizar o território do ramo interventricular anterior (RIA) ou Dg em 7 casos, da coronária direita em 7 e o da circunflexa em 18. A mortalidade hospitalar foi de $2(6 \%)$ casos, sendo ambos de causa não cardíaca. Reestudo angiográfico antes da alta hospitalar foi realizado em todos os pacientes que sobreviveram, demonstrando enxertos patentes em $96 \%(46 / 48)$. Dois enxertos exibiram espasmo moderado relacionado à ponta do cateter, um apresentou espasmo acentuadoe $e$ dois demonstraram a presença de string sign. Oito pacientes com evolução pós-operatória tardia superior a 6 meses foram submetidos a reestudo angiográfico seqũencial, com enxertos pérvios em $100 \%$, sem nenhuma anormalidade detectável. Uma paciente que apresentou string sign no pós-operatório imediato foi reestudada no $4^{2}$ mês de pós-operatório, com progressão do processo. Os resultados aqui obtidos nos permitem afirmar que a RA pode ser uma excelente opçāo para pacientes com varizes dos MMII ou ausência de veias safenas. O seguimento clínico mais prolongado nos ajudará a determinar o real valor da RA como enxerto aorto-coronário.

DESCRITORES: Miocárdio, revascularização com artéria radial, estudo clínico. Miocárdio, revascularização com artéria radial, estudo angiográfico. Miocárdio, revascularização, cirurgia.

\section{INTRODUÇÃO}

Já está bem estabelecido que a utilização da artéria torácica interna (ATI) na-cirurgia de revascularização do miocárdio proporciona melhores resultados anatômicos e funcionais imediatos e tardios, quando comparados com o emprego da veia safena $16,19,21,24$.

A utilização das duas ATI, mesmo através de suas formas mais complexas e sofisticadas, como as anastomoses seqüenciais ou enxertos em forma de $T$ ou $Y$, freqũentemente não são suficientes para a revascularização completa do miocárdio, sendo, inclusive, contra-indicada em algumas situaçōes 22, 30 .

Baseados nos excelentes resultados obtidos com as ATI, outros enxertos arteriais, como a artéria gastro-omental direita, epigástrica inferior, esplênica, intercostal suprema e gastro-omental esquerda, têm sido propostos como alternativas potencialmente superiores à utilização das veias safenas $2,5,6-7,13$, $18,27-29,33,36,37$.

O emprego da artéria radial (RA) como enxerto aorto-coronário foi inicialmente descrito por

Trabalho realizado no Serviço de Cirurgia da Santa Casa de Misericórdia de Curitiba. Curitiba, PR, Brasil.

Apresentado ao $22^{\circ}$ Congresso Nacional de Cirurgia Cardiaca. Brasilia, DF, 30 de março a $1^{2}$ de abril, 1995.

* Do Serviço de cirurgia da Santa Casa de Misericórdia de Curitiba.

Endereço para correspondência: Francisco Affonso da Costa. Rua Henrique Coelho Neto, 55. CEP 82200-120 Curitiba, PR, Brasil. Tel. [041] 253.9165 
COSTA, F. D. A.; POFFO, R.; LIMA, M. A. C.; FARACO, D. L.; SALLUM, F. S.; OLIVEIRA, E. C. C.; COSTA, I. A. - Revascularização do miocárdio com emprego da artéria radial: estudo clínico e angiográfico seqūencial. Rev. Bras. Cir. Cardiovasc., 10 (4): 165$174,1995$.

CARPENTIER et alii ${ }^{10}$, em 1973 , sendo, entretanto, logo abandonado, devido ao alto índice de oclusão precoce, causado, fundamentalmente, por espasmo e hiperplasia da íntima.

Vinte anos mais tarde, em 1992, com melhor compreensão dos mecanismos fisiopatológicos envolvidos na gênese desses eventos, ACAR et alii ${ }^{1}$ reintroduziram o seu emprego; os resultados clínicos e angiográficos obtidos foram, indiscutivelmente, bastante encorajadores.

O presente trabalho visa mostrar os nossos resultados iniciais com o emprego da RA, através da análise clínica e controle angiográfico seqüencial, tentando contribuir na determinação do real valor deste enxerto na cirurgia de revascularização do miocárdio.

\section{CASUISTICA MÉTODOS}

De $8 / 4 / 94$ a 28/2/95, 184 pacientes foram submetidos, consecutivamente, a revascularização do miocárdio (RM), isoladamente ou com procedimentos associados.

Em $32(17,3 \%)$ pacientes a artéria radial (RA) foi utilizada como um dos enxertos, geralmente como forma complementar da revascularização associada a uma ou duas artérias torácicas internas (ATI). As indicações para o emprego da RA foram a constata- ção de veias safenas de má qualidade $(n=6)$, revascularização de áreas miocárdicas parcialmente infartadas $(n=14)$ e, posteriormente, o desejo de revascularização completa com enxertos arteriais $(n=12)$.

Vinte e três $(71,8 \%)$ pacientes eram do sexo masculino e a idade variou de 41 anos a 74 anos, com média de 56,8 anos.

Os dados epidemiológicos, incluindo alguns fatores de risco para a doença coronária e características clínicas dos 32 pacientes encontram-se na Tabela 1.

\section{TÉCNICA OPERATOÓRIA}

Todos os pacientes foram submetidos, no préoperatório, à manobra de Allen para verificação da irrigação da mão através da via artéria ulnar e arcos palmares. $\mathrm{Na}$ maioria dos pacientes foi também realizada investigação através de Doppler vascular.

Em todos os pacientes foi usada sonda nasogástrica, para administração de Diltiazen nos períodos trans e pós-operatório imediato.

Paralelamente à toracotomia mediana e dissecção de uma ou ambas ATI, um dos membros da equipe cirúrgica procedeu à dissecção da RA, com incisão no antebraço desde o punho até a prega do

TABELA 1

DADOS EPIDEMIOLÓGICOS DOS 32 PACIENTES SUBMETIDOS A CIRURGIA DE REVASCULARIZAÇÃO DO MIOCÁRDIO COM A ARTÉRIA RADIAL.

\begin{tabular}{lcc}
\hline Características & Número & $\%$ \\
\hline Número de Pacientes & 32 & $(100 \%)$ \\
HAS & 14 & $(43,7 \%)$ \\
Tabagismo & 15 & $(46,8 \%)$ \\
Diabetes Melito & 2 & $(6,2 \%)$ \\
Angina grau III ou IV & 24 & $(75 \%)$ \\
Infarto Prévio & 15 & $(46,8 \%)$ \\
Lesão Arterial & & $(18,7 \%)$ \\
$\quad$ 2 vasos & 6 & $(71,8 \%)$ \\
$\quad$ tronco & 23 & $(9,3 \%)$ \\
Angioplastia Prévia & 3 & $(21,8 \%)$ \\
Revascularização Prévia do Miocárdio & 7 & $(3,1 \%)$ \\
Cirurgia de Emergência & 1 & $(3,1 \%)$ \\
\hline
\end{tabular}

HAS: Paciente com pressão arterial em repouso maior que $140 / 90 \mathrm{mmHg}$ ou fazendo uso de drogas anti-hipertensivas.

Diabetes melito: Paciente fazendo uso de insulina ou agentes hipoglicemiantes orais ou glicemia de jejum $>110 \mathrm{mg} \%$.

Angina: Graduada de acordo com a classificação de angina da New York Heart Association (NYHA).

Lesão Arterial: Lesões superiores a $70 \%$ nas artérias principais ou superiores a $50 \%$ no tronco da coronária esquerda, determinadas pela cineangiocoronariografia.

Infarto Antigo: História clínica pregressa, presença de ondas Q patológicas no ECG e/ou áreas correspondentes de acinesia na ventriculografia. 
COSTA, F. D. A.; POFFO, R.; LIMA, M. A. C.; FARACO, D. L.; SALLUM, F. S.; OLIVEIRA, E. C. C.; COSTA, I. A. - Revascularizaçāo do miocárdio com emprego da artéria radial: estudo clínico e angiográfico seqüencial. Rev. Bras. Cir. Cardiovasc., 10 (4): 165$174,1995$.

cotovelo. Após a divisão da fáscia antebraquial e exposição da RA, os ramos arteriais musculares foram divididos com eletrocautério e a RA retirada como um pedículo juntamente com as suas duas veias satélites. Procedeu-se, então, a delicada dilatação hidrostática do enxerto utilizando-se sangue heparinizado contendo solução de papaverina $(40 \mathrm{mg} / \mathrm{l})$. Em nenhuma ocasião foi realizada dilatação com probes metálicos, prevenindo-se, dessa forma, a ocorrência de lesões endoteliais.

Dessa maneira, obteve-se enxertos com aproximadamente $15 \mathrm{~cm}$ a $20 \mathrm{~cm}$ em comprimento e cujos diâmetros foram avaliados entre 2,5 a 3,5 $\mathrm{mm}$. Em nenhuma ocasião foi necessário desprezar 0 enxerto.

A dissecção das ATI e das veias safenas foi realizada de acordo com técnica previamente descrita.

Todas as operações foram realizadas com auxilio de circulação extracorpórea, oxigenadores de membrana Oxim-Macchi e hipotermia moderada de $32^{\circ} \mathrm{C}$.

A proteção miocárdica foi obtida através de cardioplegia sangüínea gelada intermitente a cada $15 \mathrm{~min}-20 \mathrm{~min}$, com reperfusão cardioplégica normotérmica de $1 \mathrm{~min}$, antes do despinçamento da aorta.

O tempo médio de pinçamento aórtico foi de 56 $\min$ (20 $\mathrm{min}$ a $75 \mathrm{~min}$ ) e o de circulação extracorpórea $101 \mathrm{~min}$ (50 $\mathrm{min}$ a $148 \mathrm{~min})$.

As anastomoses proximais da RA e ATI foram realizadas com pinçamento tartgencial da aorta ascendente, utilizando-se sutura contínua de Prolene 6-0 e as da veia safena com sutura contínua de Prolene 5-0. As anastomoses distais de RA e ATI foram com sutura contínua de Prolene $7-0$, enquanto se utilizou Prolene 6-0 para as anastomoses com veia safena.

Foram realizadas uma média de 3,28 anastomoses / paciente, sendo 1,56.com a RA, 1,34 com as ATI e $0,37 \mathrm{com}$ veia safena. A RA foi utilizada nos 32 pacientes, a ATIE em 31 , a ATID em 14 e a veia safena em 12 casos.

O local das anastomoses com a RA e suas respectivas freqüências encontram-se na Tabela 2.

Para avaliar os resultados, analisamos, retrospectivamente, as seguintes variáveis pós-operatórias:

a) mortalidade hospitalar, definida como qualquer morte até 30 dias após a operação.

b) Necessidade de drogas inotrópicas e/ou uso de balão intra-aórtico.
TABELA 2

LOCAL E FREQUENNCIA DAS ANASTOMOSES COM A ARTÉRIA RADIAL

\begin{tabular}{lc}
\hline Local & No de Casos \\
\hline RIA & 4 \\
Dg & 1 \\
Cx & 2 \\
Mg & 5 \\
Dir ou RIP & 3 \\
Dg - Mg seqüencial & 8 \\
Mg - Mg seqũencial & 3 \\
RIP - VP seqũencial & 4 \\
Dg - RIA seqüencial & 2 \\
Total & 32 \\
\hline
\end{tabular}

RIA: Ramo interventricular anterior. Dg: Diagonal. Cx, Circunflexa. Mg: Marginal. Dir: Coronária Direita, RIP: Ramo interventricular posterior. VP: Ramo ventricular posterior.

c) Ocorrência de infarto per-operatório, evidenciado pelo aparecimento de supradesnível do segmento ST ou novas ondas Q no ECG, elevação de enzimas ou comprovados por cineangiocoronariografia pós-operatória.

d) Sangramento total até a retirada dos drenos e necessidade de reoperação por sangramento.

e) Insuficiência respiratória, definida como necessidade de intubação endotraqueal por mais de $48 \mathrm{~h}$.

f) Insuficiência renal, definida pela presença de oligúria (débito urinário $<400 \mathrm{ml}$ em $24 \mathrm{~h}$ ) e elevação da creatinina sérica $>2,0 \mathrm{mg} \%$.

g) Infeç̧ão superficial e/ou profunda - foi considerada infecção superficial a presença de sinais inflamatórios locais, presença de secreção e cultura positiva. A mediastinite foi considerada nos casos de deiscência esternal associada a culturas positivas.

h) AVC, qualquer déficit neurológico novo, focal ou generalizado, detectado após a cirurgia.

i) Sinais e sintomas de isquemia da mão, assim como sintomas neurológicos nesta região no período de pós-operatório.

j) Avaliação da capacidade funcional dos pacientes.

Foram realizados estudos cineangiocoronariográficos de controle em todos os sobreviventes antes da alta hospitalar, no sentido de avaliar a real condição dos enxertos. Especial atenção foi prestada aos enxertos de RA, classificando-os em 5 categorias: a) normal, b) espasmo leve a moderado relacionado com a ponta do cateter; c) espasmo 
COSTA, F. D. A.; POFFO, R.; LIMA, M. A. C.; FARACO, D. L.; SALLUM, F. S.; OLIVEIRA, E. C. C.; COSTA, I. A. - Revascularização do miocárdio com emprego da artéria radial: estudo clínico e angiográfico seqũencial. Rev. Bras. Cir. Cardiovasc., 10(4):165$174,1995$.

acentuado relacionado com a ponta de cateter; d) string sign definido como estreitamento difuso em toda a extensão do enxerto; e) oclusão.

Em 8 pacientes com evolução pós-operatória superior a 6 meses, foi realizado reestudo cineangiocoronariográfico "tardio". Adicionalmente, uma paciente que aprsentou string sign no estudo imediato foi reestudada com 4 meses de pós-operatório.

\section{RESULTADOS}

A mortalidade hospitalar foi de $2(6,2 \%)$ casos, sendo ambos de causa não cardíaca. O primeiro óbito ocorreu em paciente de 72 anos de idade, portadora de bronquiectasia e submetida a cirurgia de emergência, e que veio a falecer com insuficiência respiratória no $8^{\circ}$ dia. O outro óbito foi decorrente de AVC comprometendo tronco cerebral, em paciente com 69 anos de idade.

Do ponto de vista hemodinâmico, a evolução foi bastante favorável, sendo que apenas 4 pacientes necessitaram de dopamina na dose de $5 \mathrm{mg} / \mathrm{kg} / \mathrm{min}$ por período maior que $24 \mathrm{~h}$.

Nenhum paciente necessitou de auxílio do balão intra-aórtico.

Ocorreu $1(3,1 \%)$ caso de infarto transoperatório, secundário a oclusão de uma ATIE anastomosada em RIA intramiocárdico.

O sangramento total no pós-operatório variou de $250 \mathrm{ml}$ a $2000 \mathrm{ml}$ (média $600 \mathrm{ml}$ ); 1 paciente foi reoperado por hemorragia, no $2^{\circ}$ dia de pós-operatório.

Dois pacientes necessitaram de intubação endotraqueal por período maior que $48 \mathrm{~h}$, na paciente que apresentou IAM transoperatório e permaneceu $56 \mathrm{~h}$ no respirador e no paciente com AVC, que veio a falecer. renal.

Não foram observados casos de insuficiência
TABELA 3

REESTUDO IMEDIATO DOS ENXERTOS

\begin{tabular}{lccc}
\hline & $\begin{array}{c}\text { Anastomoses } \\
\text { Reestudadas }\end{array}$ & $\begin{array}{c}\text { Anastomoses } \\
\text { Abertas }\end{array}$ & $\%$ \\
\hline RA & 48 & 46 & $96 \%$ \\
ATIE & 30 & 29 & $97 \%$ \\
ATID & 12 & 11 & $92 \%$ \\
Safena & 10 & 9 & $90 \%$ \\
\hline
\end{tabular}

RA: Artéria radial. ATIE: Artéria torácica interna esquerda. ATID: Artéria torácica interna direita.

Com relação à infecção, tivemos 1 caso de mediastinite por $S$. aureus em paciente que recebeu a ATI bilateral enxerto de artéria radial, e que evoluiu satisfatoriamente após cirurgia corretiva com retalhos musculares. Um segundo paciente apresentou infecção superficial da ferida operatória, sendo bem manejado com curativos locais.

Conforme já referido, 1 paciente apresentou AVC comprometendo tronco cerebral, que evoluiu para óbito no $8^{2}$ dia de pós-operatório.

Não foram observados sinais isquêmicos na mão; entretanto, 3 pacientes apresentaram disestesia transitória do polegar.

Todos os pacientes $(n=30)$ que sobreviveram à cirurgia consentiram em realizar estudo cineangiocoronariográfico antes da alta hospitalar. No total foram reestudadas 48 anastomoses de artéria radial, 42 de artéria torácica interna ( 30 da ATIE e 12 da ATID) e 10 anastomoses com a safena. A condição imediata dos enxertos está relacionada na Tabela 3.

A análise mais detalhada das 48 anastomoses de artéria radial reestudadas no período de pósoperatório imediato está na Tabela 4.

Nos 5 pacientes que apresentaram espasmo localizado ou difuso, foi realizada infusão de nitroglicerina intracoronária, obtendo-se melhora nos 2 casos que apresentavam espasmo moderado. Nos outros 3 pacientes não houve resposta à infusão de vasodilatadores.

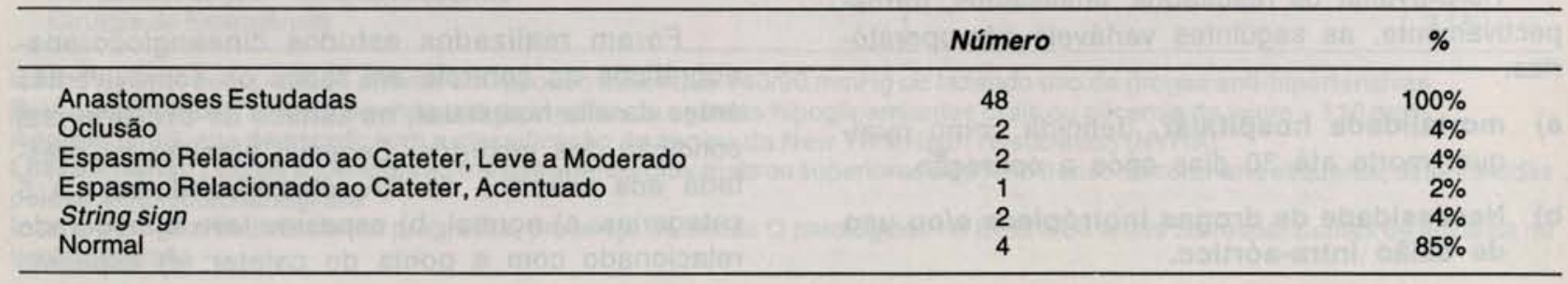


COSTA, F. D. A.; POFFO, R.; LIMA, M. A. C.; FARACO, D. L.; SALLUM, F. S.; OLIVEIRA, E. C. C.; COSTA, I. A. - Revascularização do miocárdio com emprego da artéria radial: estudo clínico e angiográfico seqüencial. Rev. Bras. Cir. Cardiovasc., 10(4): 165$174,1995$.

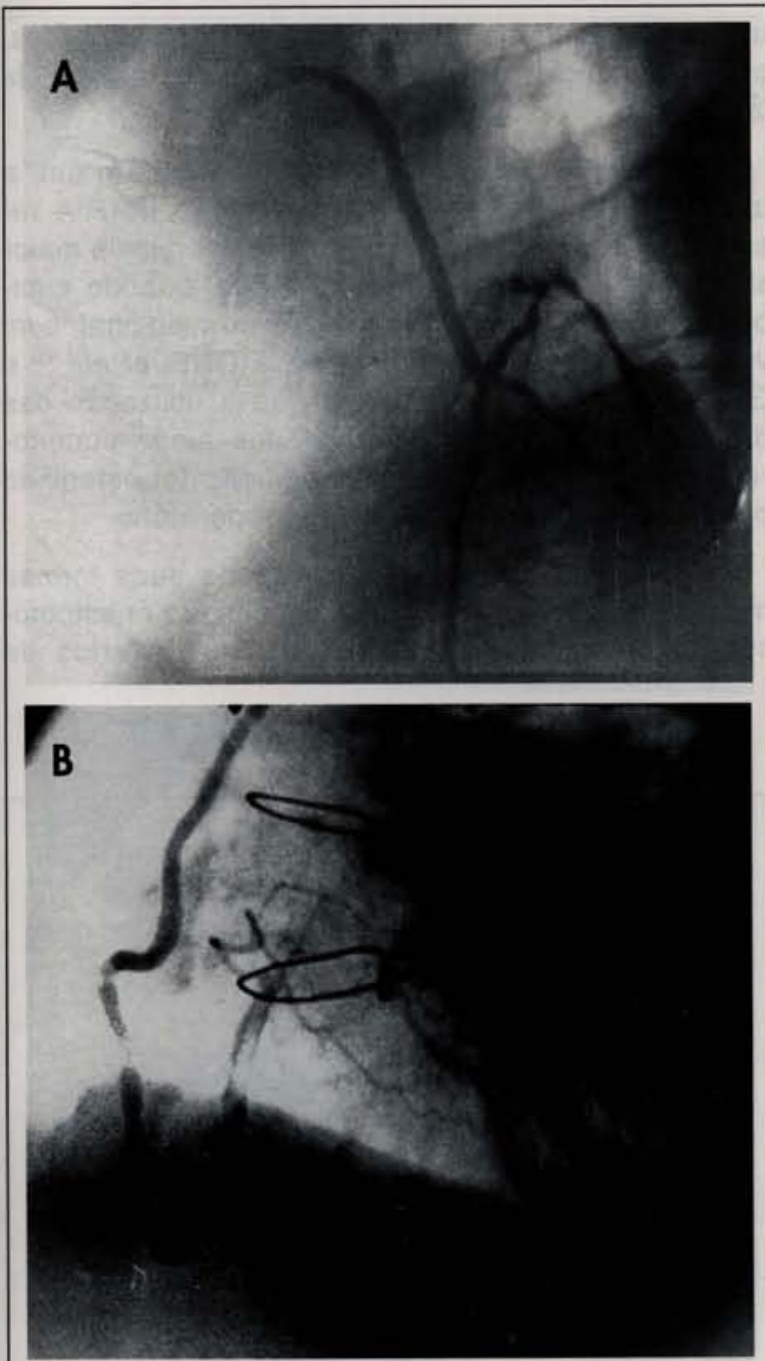

Exemplos de estudos angiográficos pós-operatórios encontram-se nas Figuras 1 e 2.

Oito pacientes com evolução superior a $6 \mathrm{me}$ ses $(6 \mathrm{~m}$ a $9 \mathrm{~m}$, média 7.4$)$ foram submetidos a estudo cineangiocoronariográfico tardio, no sentido de avaliar a presença de espasmos ou sinais de hiperplasia da intima. Nos 8 reestudos, todas as RA apresentavam-se amplamente pérvias, sem qualquer anormalidade. Um dos pacientes reestudados, e que apresentava espasmo moderado no pós-operatório imediato, demonstrou enxerto normal do reestudo tardio. Exemplos de reestudos seqüenciais encontram-se nas Figuras 3 e 4.

Uma das pacientes que apresentou string sign no reestudo imediato foi reestudada no $4^{\circ}$ mês de
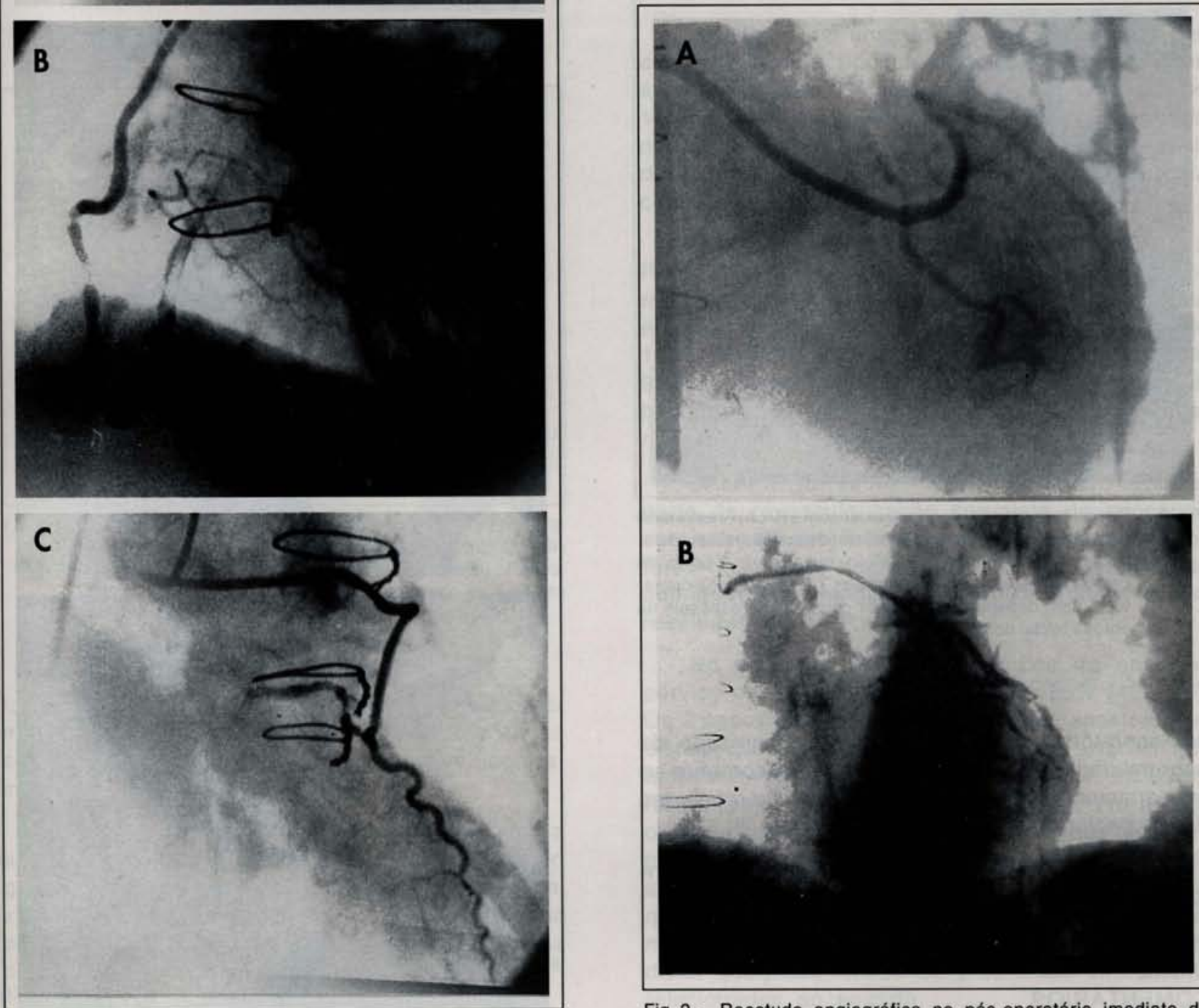

Fig. 1 - Reestudo angiográfico no pós-operatório imediato de anastomoses com a artéria radial. A) Anastomose da RA para o RIA;B) Anastomose da RA para a CD;C) Anastomose da RA para a Mg. $\mathrm{O}$ aspecto angiográfico de todos esses enxertos foi considerado normal.

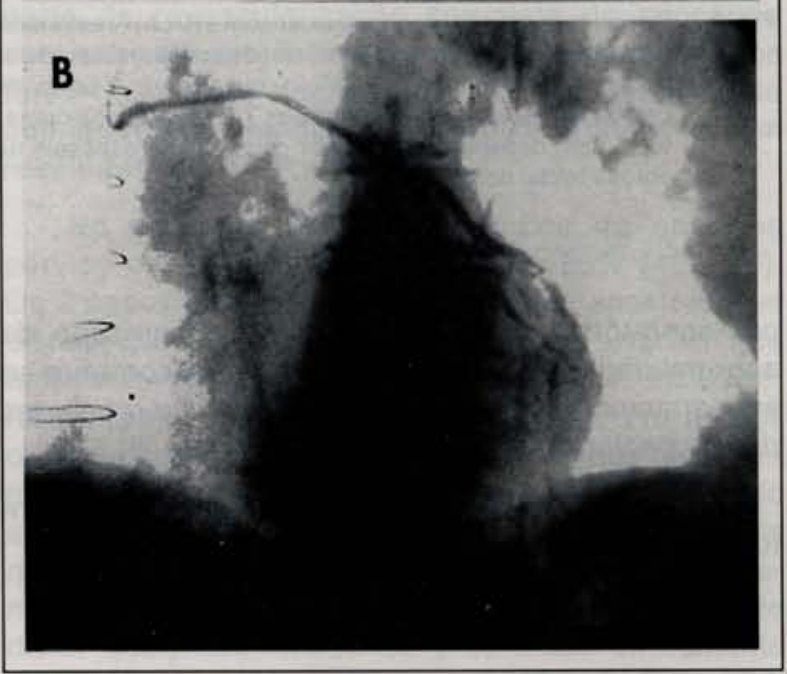

Fig. 2 - Reestudo angiográfico no pós-operatório imediato de anastomoses seqũenciais com a artéria radial.A) Anastomose da RA de forma seqũencial para $\mathrm{Dg}$ - Mg;B) Anastomose da RA de forma sequéencial para $\mathrm{Mg}-\mathrm{Mg}$. O comprimento dos enxertos de RA freqũentemente é suficiente para feitura de anastomoses deste tipo. 
COSTA, F. D. A.; POFFO, R.; LIMA, M. A. C.; FARACO, D. L.; SALLUM, F. S.; OLIVEIRA, E. C. C.; COSTA, I. A. - Revascularização do miocárdio com emprego da artéria radial: estudo clínico e angiográfico seqũencial. Rev. Bras. Cir. Cardiovasc., 10 (4): 165$174,1995$.
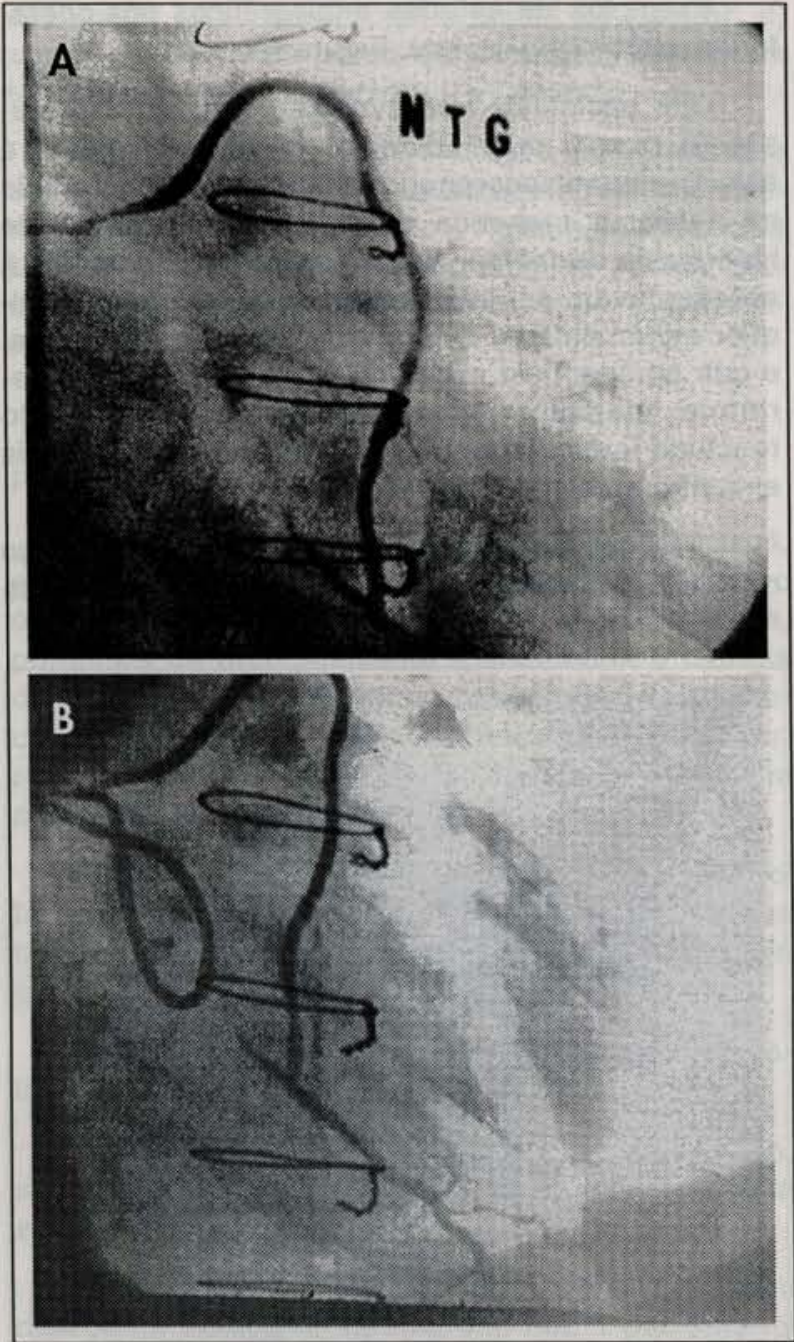

Fig. 3 - Reestudo seqũencial de anastomose coma RA.A)Anastomose para Mg no pós-operatório imediato, demonstrando moderado espasmo no início do enxerto, relacionado à ponta do cateter; B) Mesma anastomose aos 7 meses de pós-operatório, com desaparecimento por completo do espasmo na porção inicial do enxerto.

pós-operatório. Nesse caso, houve progressão da anormalidade, sendo que o enxerto encontrava-se em espasmo difuso no reestudo tardio. Os reestudos dessa paciente encontram-se na Figura 5.

Clinicamente, todos os pacientes estão assintomáticos na evolução pós-operatória tardia.

\section{COMENTÁRIOS}

Com as técnicas cirúrgicas e métodos de proteção miocárdica atualmente disponíveis, a cirurgia de RM pode ser realizada com baixos índices de morbi-mortalidade ${ }^{21}$. A maior preocupação encon- tra-se na adequada seleção dos enxertos utilizados, para a obtenção de melhores resultados funcionais tardios 19

Em 1986, LOOP et alii ${ }^{24}$ demonstraram que a utilização da ATIE na revascularização do RIA resultou em maior número de enxertos pérvios e maior sobrevida aos 10 anos de evoluçäo, quando comparado com a revascularização convencinal com veia safena. Mais recentemente, FIORE et alii ${ }^{16} \mathrm{e}$ GALBUT et alii ${ }^{19}$ concluíram que a utilização das duas ATI pode fornecer resultados ainda superiores, quando o período de observação foi estendido para 15 anos - 17 anos de pós-operatório.

As duas ATI, mesmo através de suas formas mais complexas e sofisticadas, incluindo anastomoses seqüenciais," enxertos livres ou enxertos da
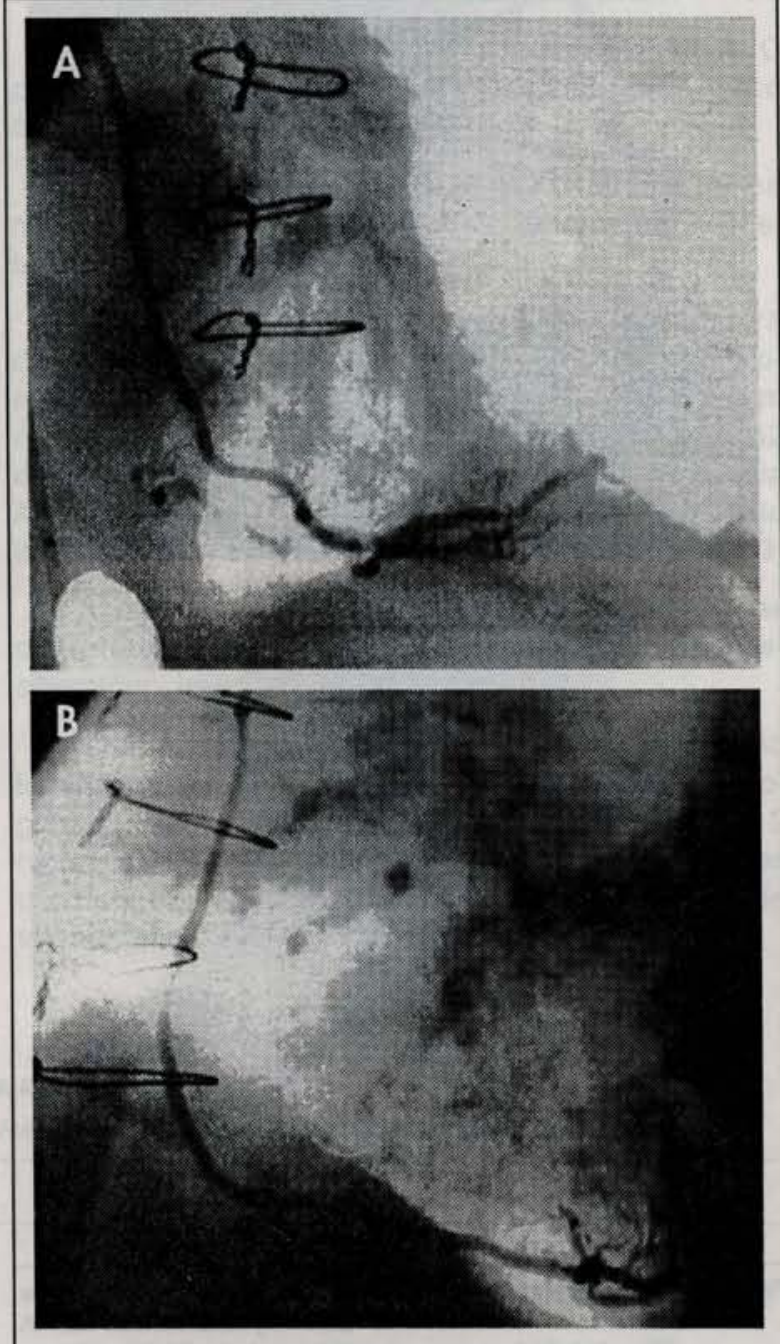

Fig. 4- Reestudo seqüencial de anastomose coma RA.A)Anastomose para o RIP no pós-operatório imediato;B) Mesma anastomose aos 6 meses de pós-operatório. 
COSTA, F. D. A.; POFFO, R.; LIMA, M. A. C.; FARACO, D. L.; SALLUM, F. S.; OLIVEIRA, E. C. C.; COSTA, I. A. - Revascularização do miocárdio com emprego da artéria radial: estudo clínico e angiográfico seqüencial. Rev. Bras. Cir. Cardiovasc., 10 (4): 165$174,1995$.

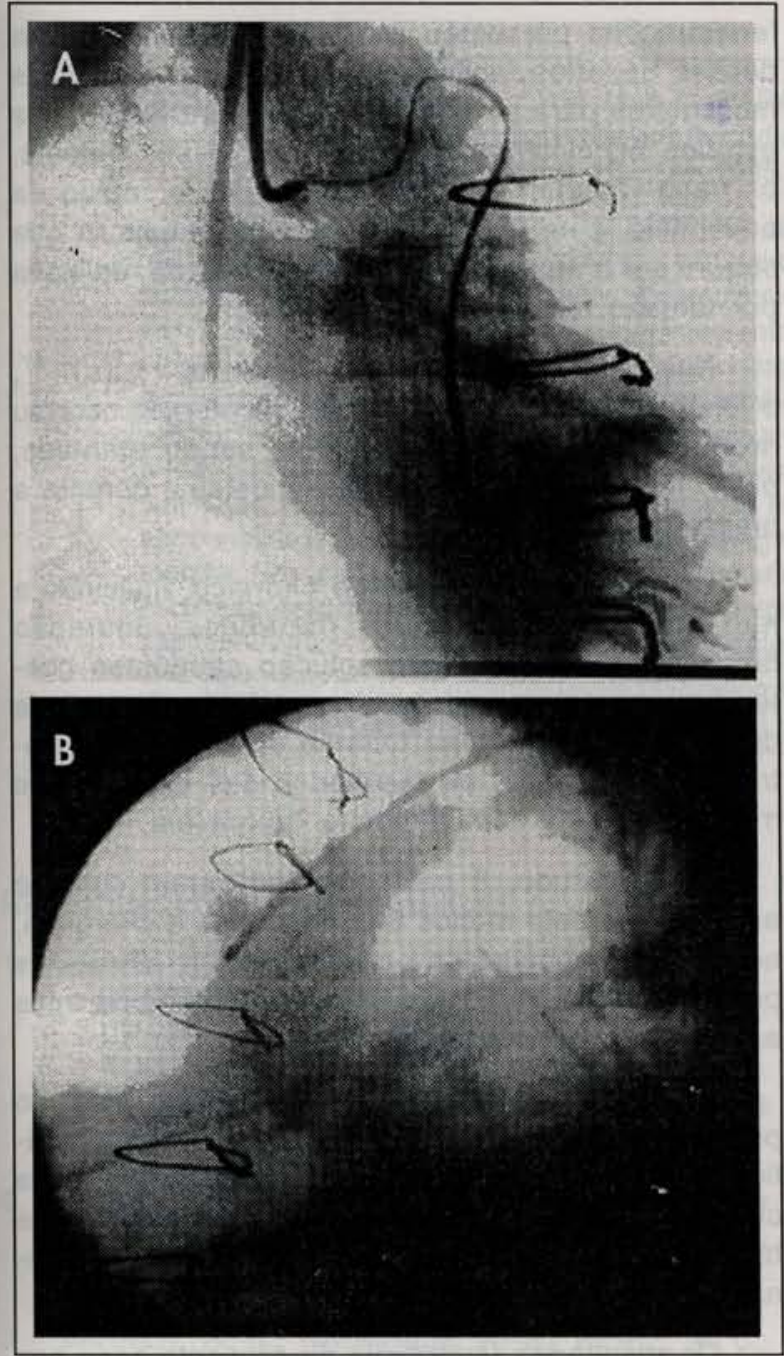

Fig. 5 - Reestudo seqüencial de anastomose da RA para o ramo Mg. A) Pós-operatório imediato demonstrando string sign; B) Reestudo no $4^{2}$ mês de pós-operatório mostrando progressão do espasmo, entretanto o enxerto ainda se encontra pérvio.

torácica interna em forma de $T$ ou de $Y$, freqüentemente não são suficientes para a revascularização completa do miocárdio, sendo, inclusive, contraindicadas em algumas situações 22, 30 .

Assim sendo, outros enxertos arteriais como a artéria gastro-omental direita, epigástrica inferior, gastro-omental esquerda, esplênica e intercostal suprema têm sido propostas como alternativas potencialmente superiores à utilização da veia safena para se complementar a cirurgia de $\mathrm{RM} 2,3,6,7,8$, $13,18,27-29,33,36,37$

A utilização da RA como enxerto aorto-coronário foi descrita, inicialmente, por CARPENTIER et alii ${ }^{10}$, em 1973. Os autores relataram a sua utilização em 30 pacientes, obtendo $100 \%$ de enxertos pérvios dos comprovados por estudos angiográficos realizados entre o $1^{\circ}$ mês e $10^{\circ}$ mês de pós-operatório. Dois anos mais tarde, os mesmos autores contraindicaram a utilização da RA, pois observaram índices de oclusão de $35 \%$ no período subseqüente de avaliação ${ }^{9}$.

Experiências desfavoráveis semelhantes foram referidas por outros autores. CURTIS et alii ${ }^{14}$ empregaram a RA como enxerto aorto-coronário em 79 pacientes, dos quais 34 foram reestudados entre o $6^{\circ}$ mês e $12^{\circ}$ mês de pós-operatório, encontrando 11 casos de oclusão, 11 com estenose importante e apenas 12 enxertos com aspecto angiográfico normal. FISK et alii ${ }^{17}$ tiveram índice de oclusão de $50 \%$ em pacientes reestudados até o $6^{\circ}$ mês de evolução. Resultados similares foram observados por EDWARDS 15.

Entretanto, no final da década de 80, ACAR et alii 1 tiveram a oportunidade de rever estudos angiográficos de RA implantadas por períodos de 14 anos a 17 anos, e cujo aspecto foi considerado normal. Esse fato fez com que os autores reconsiderassem a RA como um enxerto adequado na cirurgia de RM.

Entre 1989 e 1991, ACAR et alii ${ }^{1}$ utilizaram a RA em 104 pacientes, fazendo controle cineangiocoronariográfico nos 50 casos iniciais. O índice de enxertos pérvios no pós-operatório imediato foi de $100 \%$ e, no reestudo tardio ( 9,2 meses), foi de $93,5 \%$. Esses resultados foram, indubitavelmente, bastante superiores àqueles encontrados em experiências anteriores.

CALAFIORE et alii ${ }^{8}$ empregaram a RA como parte da revascularização completa do miocárdio com enxertos arteriais. Vinte e seis desses enxertos foram estudados angiograficamente no período de pós-operatório imediato com $100 \%$ pérvios e, em reestudos "tardios", de $94,1 \%$.

No presente trabalho, o indice de enxertos pérvios em período imediato foi de $96 \%$ (46/48). Os 2 casos de oclusão ocorreram nas anastomoses distais de enxertos sequenciais, em artérias que apresentavam mau leito distal (em uma, inclusive, foi realizada extensa endarterectomia). Em ambos os casos, as anastomoses látero-laterais estavam pérvias e os enxertos apresentavam aspecto angiográfico normal. Assim, é bastante sugestivo que as duas oclusões foram devidas ou a problemas técnicos nas anastomoses, ou por falta de leito distal adequado, antes de problemas diretamente relacionados ao enxerto.

As características histológicas da RA são bastante distintas da ATI; esta apresenta uma fina camada média $(330 \mu \mathrm{m})$ constituída basicamente de fibras elásticas, enquanto na RA essa camada 
COSTA, F. D. A.; POFFO, R.; LIMA, M. A. C.; FARACO, D. L.; SALLUM, F. S.; OLIVEIRA, E. C. C.; COSTA, I. A. - Revascularização do miocárdio com emprego da artéria radial: estudo clínico e angiográfico seqũencial. Rev. Bras. Cir. Cardiovasc., 10 (4): 165$174,1995$.

é espessa $(500 \mu \mathrm{m})$ e formada essencialmente por fibras musculares 36 .

Essas diferenças podem, pelo menos parcialmente, explicar a maior propensão da RA a fenômenos de espasmo ${ }^{34}$.

Enxertos arteriais, quando utilizados de forma livre, podem estar associados à maior incidência de espasmos do que quando empregados in situ. MASSA et alii ${ }^{26}$ demonstraram, experimentalmente, que a enervação de enxertos arteriais interfere, de maneira significativa em seu tônus vasomotor e em suas respostas contráteis, a diversos estímulos químicos, pois a função dos receptores @ - adrenérgicos pós-juncionais fica alterada. Esse fenômeno pode explicar a presença de string sign observado em alguns casos de enxertos arteriais livres ralizados com $\mathrm{ATI}$, artéria gastro-omental e artéria radial $1,26,27,35$.

A utilização de papaverina e bloqueadores dos canais de cálcio, apesar de efetiva, não eliminou completamente a ocorrência de espasmos na RA, conforme demonstram a experiência de ACAR et alii ${ }^{1}$ e o presente estudo.

Os fatores responsáveis pelos excelentes resultados com a ATI são múltiplos, incluindo o fato de ser um enxerto arterial, ter nutrição predominantemente através da luz do vaso, riqueza de drenagem linfática e produção de prostaciclinas e fator relaxante derivado do endotélio. Até que ponto a RA preencherá todos esses critérios ainda não está definido.

O maior questionamento com relação à utilização dos enxertos de RA refere-se à ocorrência de hiperplasia da íntima e, conseqüentemente, à disfunção do enxerto ${ }^{12}$. VAN SONS et alii 35,36 referem que a RA, quando utilizada como enxerto livre, estaria propensa a desenvolver hiperplasia da íntima, pois a sua espessa camada média poderia estar mais vulnerável à isquemia secundária à interrupção dos vasa-vasorum. Adicionalmente, as fenestrações presentes na lâmina elástica interna desses enxertos facilitariam a migração de células musculares para a região da íntima. Entretanto, nos estudos histológicos realizados por esses autores, os vasa-vasorum estavam confinados à região da adventícia, sem penetrar na camada média, o que sugere ser a nutrição desta última camada realizada por difusão através da lùz do vaso.

Nas experiências anteriores com a RA 10, 14, 15, 17 , o fenômeno de hiperplasia intimal acelerado ocorreu nos meses iniciais de evolução, sendo atribuído, pelo menos em parte, à lesão endotelial durante a preparação do enxerto 12 .

Com adequado manuseio cirúrgico, incluindo a não utilização de probes metálicos, dilatação hidrostática delicada com solução sangüínea contendo papaverina, uso de bloqueadores dos canais de cálcio e evitando-se tocar o endotélio com instrumental cirúrgico, os resultados das experiências mais recentes foram bastante favoráveis.

Neste estudo, 8 pacientes realizaram controle angiográfico pelo menos 6 meses após a cirurgia $e$, em todos, o aspecto do enxerto foi satisfatório; nos parece improvável que venham a se deteriorar após esse tempo de evolução.

Entretanto, uma paciente com "string sign" no pós-operatório imediato apresentou piora angiográfica em reestudo no $4^{\circ}$ mês de pós-operatório. Esse caso isolado indica que o problema do espasmo e hiperplasia da íntima não está totalmente solucionado.

Os resultados favoráveis da presente série nos permitem afirmar que a RA pode ser utilizada com sucesso na cirurgia de RM, sendo excelente opção em pacientes com ausência de veia safena ou com varizes dos MMII. Somente através de seguimento clínico mais prolongado poderemos determinar o seu real valor como enxerto aorto-coronário. 
COSTA, F. D. A.; POFFO, R.; LIMA, M. A. C.; FARACO, D. L.; SALLUM, F. S.; OLIVEIRA, E. C. C.; COSTA, I. A. - Revascularização do miocárdio com emprego da artéria radial: estudo clínico e angiográfico seqũencial. Rev. Bras. Cir. Cardiovasc., 10 (4): 165174,1995

RBCCV 44205-274

COSTA, F. D. A.; POFFO, R.; LIMA, M. A. C.; FARACO, D. L.; SALLUM, F. S.; OLIVEIRA, E. C. C.; COSTA,

I. A. - Myocardial revascularization with the radial artery: clinical and sequential angiographic study. Rev. Bras. Cir. Cardiovasc., 10 (4): 165-174, 1995.

ABSTRACT: From 4/8/94 thru 2/28/95, 32 patients were submitted to myocardial revascularization utilizing the radial artery graft (RA) as part of the procedure. This represented $17.3 \%$ of our coronary bypass surgery experience during that period. Twenty three patients were male and mean age was 56.8 years (4174). The RA was used to graft the anterior interventricular area in 7 cases, right coronary artery in 7 cases and circunflex in 18 cases. Two patients died, for a hospital mortality of $6 \%$, being of non-cardiac causes in both. Angiographyc control was done in all hospital survivors before discharge, revealing $96 \%$ of graft patency rate (46/48). However, there were two cases of moderate spasm related to catheter tip, one case of severe spasm and two grafts exibit string sign. Eight patients with follow-up time greater than 6 months (mean 7.4) were submitted to sequential angiographyc control, showing $100 \%$ patency rate, with no detectable visual abnormalities. One patient which showed "string sign" at her first angiographyc study was restudied at 4 months. Her graft actually showed progression of the abnormality, being, however, still patent. The results herein obtained allow us to conclude that the RA might be an excelent option for patients with varicose or absent saphenous vein. Longer follow-up studies will determine the real value of the RA as aortocoronary grafts.

DESCRIPTORS: Myocardial revascularization with radial artery, clinical study. Myocardial revascularization with radial artery, angiographic study. Myocardial revascularization, surgery.

\section{REFERÊNCIAS BIBLIOGRÁFICAS}

1 ACAR, C.; JEBARA, V. A.; PORTOGHESE, M.; BEYSSEN, B.; PAGNY, J. Y.; GRARE, P.; CHACHQUES, J. C.; FABIANI, J.; DELOCHE, A.; GUERMONPREZ, J. L.; CARPENTIER, A. F. Revival of the radial artery for coronary artery bypass grafting. Ann. Thorac. Surg., 54: 652-660, 1992.

2 ATTUM, A. A. - The use of the gastroepiploic artery for coronary artery bypass graft: another alternative. Texas Heart Inst. J., 14: 289-292, 1987.

3 BARNER, H. B.; NAUNHEIM, K. Š.; FIORE, A. C.; FISCHER, V. W.; HARRIS, H. H. - Use of the inferior epigastric artery as a free graft for myocardial revascularization. Ann. Thorac. Surg., 52: 429-437, 1991.

BOERBOOM, L. E.; OLINGER, G. N.; BONCHEK, L. I.; GUNAY, I. I.; KISSEBAH, A. H.; RODRIGUEZ, E. R.; FERRANS, V. J. - The relative influence of arterial pressure versus intraoperative distention on lipid accumulation in primate vein bypass grafts. J. Thorac. Cardiovasc. Surg., 90: 756-764, 1985.

BOURASSA, M. G.; FISHER, L. D.; CAMPEAU, L.; GILLESPIE, M. J.; MCCONNEY, M.; LESPÉRANCE, J. - Long-term fate of bypass grafts: the Coronary Artery Surgery Study (CASS) and Montreal Heart Institute experiences. Circulation, 72 (Supl. 5): 71 $77,1985$.

6 BUCHE, M.; SCHOEVAERDTS, J.; LOUAGIE, Y.; SCHROEDER, E.; MARCHANDISE, B.; CHENU, P.; DION, R.; VERHELST, R.; DELOOS, M.; GONZALEZ, E.; CHALANT, C. - Use of the inferior epigastric artery for coronary bypass. J. Thorac. Cardiovasc. Surg., 103: 665-670, 1992.
7 BUFFOLO, E.; MALUF, M.; BARONE, B.; ANDRADE, J. C.; GALLUCCI, C. - Revascularização direta do miocárdio através da artéria gastro-omental esquerda. Uma nova alternativa de desvio aorto-coronário: relato de caso. Arq. Bras. Cardiol., 48: 167-171, 1987.

8 CALAFIORE, A. M.; GIAMMARCO, G. D.; LUCIANI, N.; MADDESTRA, N.; NARDO, E. D.; ANGELINI, R. Composite arterial conduits for a wider arterial myocardial revascularization. Ann. Thorac. Surg. 58: 185-190, 1994.

9 CARPENTIER, A. - Selection of coronary bypass: anatomic physiological and angiographic considerations or vein and mammary artery grafts. Discussion of GEHA, A. S.; KRONE, R. J. Mc CORMICK, J. R. J.; BAUE, A. E. J. Thorac. Cardiovasc. Surg., 70: 414-431, 1975.

10 CARPENTIER, A.; GUERMONPREZ, J. L.; DELOCHE, A.; FRECHETTE, C.; DUBOST, C. - The aorta-tocoronary radial artery bypass graft. Ann. Thorac. Surg., 16: 111-121, 1973.

11 CHAIKHOUNI, A.; CRAWFORD, F. A.; KOCHEL, P. J.; OLANOFF, L. S.; HALUSHKA, P. V. - Human internal mammary artery produces more prostacyclin than saphenous vein. J. Thorac. Cardiovasc. Surg., 92: 88-91, 1986.

12 CHIU, C. - Why do radial artery grafts for aortocoronary bypass fail?: a reappraisal. Ann. Thorac. Surg., 22: 520-523, 1976.

13 CREMER, J.; LIESMANN, T.; WIMMER-GREINECKER, G.; ABRAHAM, C.; MUGGE, A.; HAVERICH, A. - In vivo comparision of free coronary grafts using the inferior epigastric (IEA), the gastroepiploic (GEA) and 
COSTA, F. D. A.; POFFO, R.; LIMA, M. A. C.; FARACO, D. L.; SALLUM, F. S.; OLIVEIRA, E. C. C.; COSTA, I. A. - Revascularizaçāo do miocárdio com emprego da artéria radial: estudo clínico e angiográfico seqũencial. Rev. Bras. Cir. Cardiovasc., 10 (4):165$174,1995$.

the internal thoracic artery (ITA). Eur. J. Cardiothorac. Surg., 8: 240-246, 1994

CURTIS, J. J.; STONEY, W. S.; ALFORD Jr., W. C.; BURRUS, G. R.; THOMAS, C. S. - Intimal hyperplasia. Ann. Thorac. Surg., 20: p. 628-635, dez. 1975.

EDWARDS, S. - Intimal hyperplasia. Discussion of CURTIS, J. J.; STONEY, W. S.; ALFORD Jr., W. C.; BURRUS, G. R.; THOMAS, C. S. Ann. Thorac. Surg., 20: 628-635, 1975 .

FIORE, A. C.; NAUNHEIM, K. S.; DEAN, P.; KAISER, G. C.; PENNINGTON, G.; WILLMAN, V. L.; McBRIDE, L. R.; BARNER, H. B. - Results of internal thoracic artery grafting over 15 years: single versus double grafts. Ann. Thorac. Surg., 49: 202-209, 1990.

17 FISK, R. L.; BROOKS, C. H.; CALLAGHAN, J. C. DVORKIN, J. - Experience with the radial artery graft for coronary artery bypass. Ann. Thorac. Surg., 21: 513-518, 1976.

FOSTER, E. D. \& KRANC, M. A. T. - Alternative conduits for aortocoronary bypass grafting. Circulation, 79 : I-34-I-39, 1989.

GALBUT, D. L.; TRAAD, E. A.; DORMAN, M. J.; DeWITT, P. L.; LARSEN, P. B.; KURLANSKY, P. A.; BUTTON J. H.; ALLY, J. M.; GENTSCH, T. O. - Seventeenyear experience with bilateral internal mammary artery grafts. Ann. Thorac. Surg., 49: 195-201, 1990.

KAMIENSKI, R. W. \& BARNES, R. W. - Critique of the allen test for continuity of the palmar arch assessed by doppler ultrasound. Surg. Gynecol. Obstet., 142: 861-864, 1976.

21 KIRKLIN, J. W.; NAFTEL, D. C.; BLACKSTONE, E. H, POHOST, G. M. - Comment summary of a consensus concerning beat and ischemic events after coronary artery bypass grafting. Circulation, 79: 81-91, 1989.

KOUCHOUKOS, N. T.; WAREING, T. H.; MURPHY, S. F.; PELATE, C.; MARSHALL, W. G. - Risks of bilateral internal mammary artery bypass grafting. Ann. Thorac. Surg., 49: 210-219, 1990.

23 LOOP, F. D.; LYTLE, B. W.; COSGROVE, D. M.; GOLDING, L. A. R.; TAYLOR, P. C.; STEWART, R. W. - Free (aorto-coronary) internal mammary artery graft. J. Thorac. Cardiovasc. Surg., 92: 827-831, 1986.

24 LOOP, F. D.; LYTLE, B. W.; COSGROVE, D. M.; STEWART, R. W.; COORMASTIC, M.; WILLIAMS, G. W.; GOLDING, L. A. R.; GILL, C. G.; TAYLOR, P. C.; SHELDON, W. C.; PROUDFIT, W. - Influence of the internal mammary artery graft on 10 - year survival and other cardiac events. N. Engl. J. Med., 314: $1-6,1986$

LÜSCHER, T. F.; DIEDERICH, D.; SIEBENMANN, R.;
LEHMANN, K.; STULZ, P.; SEGESSER, L.; YANG, Z.; TURINA, M.; GRADEL, E.; WEBER, E.; BÜCHLER, F. R. - Difference between endotheliumdependent relaxation in arterial and in venous coronary bypass grafts. N. Engl. J. Med., 319: 462 $467,1988$.

MASSA, G.; JOHANSSON, S.; KIMBLAD, P.; SJOBERG, T.; STEEN, S. - Might free arterial grafts fail due to spasm? Ann. Thorac. Surg., 51: 94-101, 1991.

MILLS, N. L. \& EVERSON, C. T. - Right gastroepiploic artery: a third arterial conduit for coronary artery bypass. Ann. Thorac. Surg., 47: 706-719, 1989.

MILLS, N. L. \& EVERSON, C. T. - Technical considerations for use of the gastroepiploic artery for coronary artery surgery. J. Cardiovasc. Surg., 4: 1 9, 1989.

PYM, J.; BROWN, P. M.; CHARRETTE, E. J. P.; PARKER, J. O.; WEST, R. O. - Gastroepiploiccoronary anastomosis. J. Thorac. Cardiovasc. Surg., 94: 256-259, 1987.

SAUVAGE, L. R.; WU, H.; KOWALSKY, T. E.; DAVIS, C. C.; SMITH, J. C.; RITTENHOUSE, E. A.; HALL, D. G.; MANSFIELD, P. B.; MATHISEN, S. R.; USUI, Y.; GOFF, S. G. - Healing basis and surgical techniques for complete revascularization of the left ventricle using only the internal mammary arteries. Ann. Thorac. Surg., 42: 449-465, 1986.

1 SIMS, F. H. - The pathology of the internal thoracic artery and its contribution to the study of atherosclerosis. Tokio, Igaku-shoin, 1991. p. 18-62.

SONS, H. J.; GODECHARDT, E.; KUNERT, J.; LOSSE, B.; BIRCKS, W. - Internal thoracic artery: prevalence of atherosclerotic changes. J. Thorac. Cardiovasc. Surg., 106: 1192-1195, 1993.

3 SUMA, H.; FUKUMOTO, H.; TAKEUCHI, A. - Coronary artery bypass grafting by utilizing in situ right gastroepiploic artery: basic study and clinical application. Ann. Thorac. Surg., 44: 394-397, 1987.

4 VAN SON, J. A. M. - Revival of the radial artery for coronary artery bypass grafting: l'histoire se repete. Ann. Thorac. Surg., 55: 1593-1600, 1993. (Carta ao Editor).

5 VAN SON, J. A. M. - Spasm in free arterial grafts. Ann. Thorac. Surg., 52, 396-900, 1991. (Carta ao Editor).

6 VAN SON, J. A. M.; SMEDTS, F.; VICENT, J. G.; LIER, H. J. J.; KUBAT, K. - Comparative anatomic studies of various arterial conduits for myocardial revascularization. J. Thorac. Cardiovasc. Surg., 99: 703$707,1990$.

7 WAHBA, A.; OFFERDAL, K.; SOMMOGGY, S.; BIRNBAUM, D. E. - The morphology of the inferior epigastric artery has implications on its use as a conduit for myocardial revascularization. Eur. J. Cardiothorac. Surg., 8: 236-239, 1994. 